\title{
The comparative plastome analysis of twelve Allium species: adaptation to shaded environments could be accompanied by the complete loss function of the NDH genes
}

\author{
Speranskaya A.S. ${ }^{1 *}$, Belenikin M.S. ${ }^{2}$, Konorov E.A. ${ }^{3,4}$, Kuptsov S.V. ${ }^{1}$, Antipin M.I. ${ }^{1}$, \\ Logacheva M.D. ${ }^{5}$, Omelchenko D.O. ${ }^{1,6}$, Krinitsina A.A. ${ }^{1,7}$ \\ ${ }^{1}$ Lomonosov Moscow State University, Moscow, Russia \\ ${ }^{2}$ Moscow Institute of Physics and Technology, Dolgoprudny, Russia \\ ${ }^{3}$ Vavilov Institute of General Genetics, RAS, Moscow, Russia \\ ${ }^{4}$ V.M. Gorbatov Federal Research Center for Food Systems, RAS, Moscow, Russia \\ ${ }^{5}$ Skolkovo Institute of Science and Technology, Moscow, Russia \\ ${ }^{6}$ Institute for Information Transmission Problems, RAS, Moscow, Russia \\ ${ }^{7}$ All-Russia Research Institute of Agricultural Biotechnology, Moscow, Russia \\ *e-mail: hanna.s.939@gmail.com
}

We have analysed plastome sequences of twelve wild and cultivated Allium species (ten of them were sequenced, assembled and annotated in our lab). We have found some plastome features that are characteristic for species of three evolutionary lines of the genus, i. e. reflect evolution processes. In general the number and position of genes were similar, but $A$. paradoxum plastome sequence differed markedly from other Allium species. It had a large 4,825 bp long inversion in the SSC region and showed loss of pseudogenization of all genes encoding the NDH complex. The functional role of $n d h$ genes in plants is presumably related to adaptation to different habitats, because this complex is required to optimize photosynthesis. In $n d h$ defective plants the photosynthesis rate decreases under excess light stress conditions. Naturally $A$. paradoxum occurs in shady forests and does not grow in open areas or at forest edges. We assume that the shade-loving nature of $A$. paradoxum is caused by $n d h$ genes impairment (as a result of NDH complex interruption). No one of other analyzed Allium species demonstrated the same features, including phylogenetically related shade-loving A. ursinum .

Acknowledgements: This work was supported by the RFBR grant number 18-04-01203. 